could be replaced by more permanent and better houses. We need not worry about the waste involved in a temporary housing scheme. Our capacity for production after the War will be increased to such an extent that there will be difficulty in getting an outlet for the products. In any event, the waste of human life involved by existing slum conditions is more important than the waste of material.

As Mr. Roosevelt has pointed out, the standard of living of the masses of the people is so low that if we undertook to provide satisfactory homes and adequate food for everybody, there would be work for every man and woman seeking a job. But provision must be made for men temporarily out of employment, and every man, rich or poor, should be made to do some useful work. A National Service Corps would provide for this. The men in the Corps could be employed on national or local government work. Every man should be forced to serve for a year before he reaches twenty-one years of age. After a year's service, a man would be free to leave. He would also be free to return if he wished and could not find employment. After a man had served a number of years calculated to be sufficient to pay for the bare necessities of life on a health standard for himself and his family, he could retire and enjoy these with the same dignity and self-respect as a man drawing an endowment for which he had paid all the premiums.

This plan for adequate food, a decent home and security for every family would bring about a social revolution. Bringing food and housing among the poorest third of the population up to the standard for health would reduce disease and improve national health and physique. Poverty in the old sense of the term would be gone for ever. A great part of our social and public health services and most of our charities would become unnecessary. The unemploy. ment problem would be solved. Every family would have security for a good home. For the first time in history, the common man would enjoy economic freedom and the means of attaining his full physical and spiritual manhood.

This plan would also lay the foundation for agricultural and economic prosperity. It is estimated that in the United States the production of the protective foods, for example, milk, eggs and fruit, would need to be increased from 15 per cent in the case of some to 100 per cent in the case of others. Similar increases are required for Britain and larger increases for most other countries. The production of this additional food would call for a great expansion of agriculture. To enable agriculture to produce the food needed the great majority of farms in Britain would need to be re-equipped. The same is true of almost all other countries, including . the United States. The re-equipment of agriculture would provide an outlet for an enormous amount of industrial products. When production had reached a level which would supply human needs there will be a permanent increase in the trade in food and in the commodities exchanged for food. A food policy based on human needs would be the spear-head for agricultural and economic prosperity.

$\Lambda$ policy of providing first for human needs, as outlined here, would be so revolutionary and so beneficial that it would be wise to concentrate on it and leave all industries not directly concerned with the necessities of life to the free play of private enter. prise. This would provide for the llexibility needed for the adjustment of industry and trade to the advances of scienoe.
This plan for building the new world from the bottom upwards would give expression to our growing spiritual idealism. Every great spiritual awakening calls for sacrifice. This plan involves sacrifice. In Britain those who already enjoy the necessities of life on a health standard-and that includes the well-paid workman-would need to stand back from the national table until those worse off are served. To accomplish the world food plan, the wealthy nations must give to the poor nations food, agricultural equipment and the other things needed to provide the necessities of life, not against a loan which would sink the poorer nations in debt, but on a world-wide lease-lend plan arranged by an international financial organization, the security of which would be guaranteed by all nations, including the poorest. The poorer nations would not be encouraged to pay interest in the form of money or exports which would delay the provision of the necessities of life on a health standard for the whole of their population. When they had reached that level, they could begin to pay their debt in money or in kind. The nations would find their spiritual and economic salvation in this policy of giving.

This may seem Utopian. It is merely the policy of the good neighbour. Any ordinary citizen would give food to a neighbour needing it, demanding nothing in exchange immediately. Until Governments in their dealing with each other reach the moral standard which regulates neighbourly relations, the world will never be free from war.

The adoption of this plan would remove the feeling of uncertainty and apprehension as to what is going to happen after the War. Everyone would know what they were fighting for and working for. The people in the occupied countries in Europe and even in Germany itself would know what our peace aims are. They could be told incessantly, day and night, that when our armies invade Europe they will bring the food ships with them, and that the United Nations will continue to send food and other things needed not merely for post-war relief but until each country, including Germany, is in a position to provide the necessities of life on a health standard for the whole population. We could, in terms which would carry conviction, call upon the people of all countries to join with the armies of the free to carry through the glorious revolution for the destruction of the forces of evil, and the establishment of a new World Order in which the nations will co-operate to bring freedom from the fear of war and freedom from want to all men in all lands.

\section{QUANTUM THEORY AND DIFFUSE X-RAY REFLEXIONS}

T the article by Mr. G. D. Preston which appeared in Nature of April 4, a descriptive account was given of the theory put forward by Sir C. V. Raman in a series of papers published in the Proceedings of the Indian Academy of Sciences to explain the extra spots on Laue and other types of X-ray photographs. We here adopting a much more critical attitude.

Raman has emphasized the fact that when X-rays are scattered by a vibrating system, the scattered beam contains components of changed frequency. It is not, however, made clear either in the Symposium or in Preston's account of it that this is a consequence 
both of classical and of quantum theory. From the classical point of view it is the Doppler effect for the reflexion of X-rays by waves of atomic vibrations; in quantum language it is the transfer of energy from a photon to an acoustical quantum, or vice versa. This frequency shift has long been acknowledged by the thermal theory. Since the frequency changes are much too small to be observed, they are not of practical significance except in so far as they are intimately connected with the incoherence of the scattered rays, which is an essential feature of the phenomenon.

Raman also gives the impression (which is not refuted by Preston) that his theory is a "quantum" theory and that the thermal theory is a "classical" one. The exact opposite is the case. As Preston remarks, Raman's presentation is obscure, but it is clear enough that at no stage (except when he introduces the effect of temperature change) does Raman make use of quantum mechanismas at all. $\mathrm{He}$ does not attempt, for example, to calculate the intensity with the help of transition probabilities. The thermal theory, on the other hand, although originally presented, nineteen years ago, in a semiclassical form, has since been given a strictly quantummechanical basis. The facts are that Raman's "excited vibrations" must exist (either from a classical or a quantum point of view), but that their contribution to the scattering is extremely small as compared with the effect of the thermal vibrations.

All the experiments described by Raman and his colleagues are in entire accordance with the thermal theory, but experiments on metals and on the "forbidden" reflexions of diamond, which he did not attempt, definitely prove that his theory cannot account for the facts. We have outlined some of our arguments in the following communications (written before we had read Mr. Preston's account) and have elaborated them in papers to be published elsewhere.

M. B.

K. L. and H. S.

Sir C. V. Raman's theory of the extra spots on Laue photographs having now been published in detail, I wish to deal with the theoretical aspect, as I believe it is now possible to show exactly where Reman's statements are incorrect.

(1) Raman assumes that the extra spots are due not to the acoustical (low-frequency) branch of the vibrational spectrum but to monochromatic infra-red vibrations.

This assumption contradicts the results of lattice dynamics, which show that the elastic spectrum consista of different branches, each of $N$ vibrations ( $N$ number of unit cells), uniformly distributed in reciprocal space (co-ordinates: components of wave vector q). In order to justify this contradiction, Raman points out that lattice theory uses the method of the 'cyclic lattice' (replacing the boundary conditions by the postulate of periodicity in a parallelepiped of $N$ cells), a method which he says is not proved but arbitrarily invented to simplify the mathematical treatment. As a matter of fact, it is not proved, apart from a simple example (chain of equal particles) and a limiting case (continuous medium). These cases show, however, that the cyclic method is not arbitrary, but mathematically reasonable; and it is also well confirmed by its applications (for example, conductivity of metals). As Raman attacks the validity of the method, in particular for the optical branches, I have rigorously solved the problem of a finite di-atomic one-dimensional lattice (chain of two alternating particles); the result is, of course, uniform distribution of the frequencies in both branches and the same law for their dependence on wave number as given by the cyclic method.

The intensity of X-ray scattering of all branches will be incoherent and proportional to $N$. Raman's contention that the scattering due to an excited infra-red vibration will be coherent and proportional to $N^{2}$ has no theoretical justification whatever.

(2) Bisheshwar Dayal attempts to support Raman's assumption of monochromatic infra-red vibrations by calculations about the specific heat of metals, which he contends cannot be represented by a Debye function but needs additional terms of the Einstein type. This suggestion is not new ; it was given in my book "Dynamik der Kristallgitter", published in 1915 (formula 200 , p. 77), where I showed that the optical branches of the lattice oscillations are narrow bands which for the calculation of specific heat can be approximately replaced by one frequency. It is clear that no support for Raman's hypothesis can be obtained from a formula previously derived from lattice dynamics.

(3) Raman considers the sharpness of the lines observed in the optical Raman spectrum of crystals as a proof of the monochromatic character of the infra-red vibrations. It is not. Lattice theory explains it as a consequence of the optical selection rule: emission of light waves will not occur for every case of resciance, since the electric moments of the different cells of the crystal are in general out of phase; it is restricted to long waves for which the phases in a large group of cells are equal, and this corresponds to an extremely small region of the reciprocal space near the point $q=0$. To each 'optical branch' of frequency there belongs only one sharp 'optical resonance'. (I regret if the term 'optical branch' has led to this misunderstanding; but the facts are clearly stated in several publications.)

(4) Raman's proof that the acoustical branch cannot produce sharp spots is, shortly, the following : The scattering is proportional to the mean square of the amplitude of vibration, $\overline{\xi^{2}}$. On the other hand, the mean potential energy of an oscillator of angular frequency $\omega, \frac{1}{2} \omega^{2} \bar{\xi}^{2}$, is equal to half the mean total energy $\frac{1}{2} k T, \quad(\hbar \omega<<k T)$, hence $\overline{\xi^{2}}=k T / \omega^{2}$. Raman replaces $\omega$ by $2 \pi c / \lambda$, where $\lambda$ is the wavelength of the elastic wave, $c$ the velocity of sound. Thus he obtains the scattering of the acoustical branch proportional to $k T \lambda^{2}$, which is a smooth function and cannot give rise to spots.

The fallacy of this argument lies in the assumption that $c$ is a constant. It is not. In fact, the relation betweien $\lambda$ and $\omega$ is involved. $\omega$ is a periodic function of the components of the wave vector $q$ (length $q=\mathrm{I} / \lambda)$. Consider as illustration the oscillations of a set of parallel equidistant planes in the lattice (spacing $a$ ) which will be roughly the same as that of a chain of equidistant mass points (linear lattice); it is well known that the frequency in this case is $\omega=\omega_{0}|\sin (\pi a / \lambda)|=\omega_{0}|\sin (\pi q / b)|$, where $b=1 / a$ is the lattice constant of the reciprocal lattice. The dynamical theory of the scattering leads to the same result as de Broglie's quantum condition: the intensity is enhanced if the difference of the momentum vectors for the incident and reflected photon is equal to the momentum of the acoustical quantum, or $2 Q \sin \delta=q$, where $Q=1 / \Lambda$ is the wave number of the X-ray. (As the left-hand term may have any 